\title{
Metallographic Preparation of Self-Reacting Friction Stir Welded 2195/2219 Aluminum Alloys For Electron Backscatter Diffraction
}

\author{
J.E. Martinez, L.B. Johannes, D.J. Rybicki, J.D. Figert, J.A. Ventura \\ NASA Johnson Space Center, Structural Engineering Division \\ 2101 NASA Parkway, Houston, TX 77058
}

Light-weight aluminum alloys are needed for structural load bearing components for future manned and unmanned space vehicles. An alloy of interest is 2195 aluminum-copper-lithium (Weldalite 049). 2195 has been used for the external tanks on the space shuttle and was joined utilizing friction stir welding. However, 2195 has not been characterized with electron backscatter diffraction (EBSD) utilizing the latest and most promising joining technique - selfreacting friction stir welding (SR-FSW).

Numerous parameters during the SR-FSW process can be altered, all of which affect the microstructure along the parent and weld nugget regions. Feed rate, spindle speed, load, tool shape, and tool position relative to the plates being welded all affect the quality of weld by reshaping the grain size and redistributing the alloying elements in the weld region.

Mono and bi-metallic SR-FSW consisting of 2195/2195 and 2219/2195 aluminum alloys were evaluated for refining the various parameters listed above. Grain size and local grain misorientation are the primary micro-structural features of interest. Optical microscopy does an adequate job of evaluating the grain size along the weld region but cannot quantitatively determine crystallographic orientation. EBSD is an excellent technique for evaluating texture. Since EBSD patterns are generated from the first few surface atom layers, sample prep for EBSD is significantly more stringent than optical microscopy.

Samples were prepared using various mechanical and vibropolishing techniques to determine which produced the surface finish with least damage, thereby yielding the highest pattern quality maps. Several combinations of $\mathrm{SiC}$ paper and diamond substrates were used during the fine grinding and polishing steps $[1,2]$. After final polish, each sample was lightly etched using Keller's reagent to remove the superficial smear layer and to expose grain boundaries [3]. Samples were then imaged and pre-screened using differential interference contrast (DIC) microscopy prior to performing EBSD.

The as-polished surface appeared scratch free but once etched and viewed with DIC illumination, small scratches became visible. Samples were subsequently vibropolished in 30-minute increments to reduce scratches and to smoothen the etched surface (FIG. 1). Surface quality improved with increasing vibropolishing; but overexposure to vibropolishing resulted in pitting and colloidal silica embedding into the sample.

Figure 2 shows a typical SR-FSW in cross section. The advancing side indicates where the tool enters the first plate. The retreating side is where the tool exits the second plate being joined. The weld nugget clearly exhibits non-uniform mixing. 
EBSD maps performed on samples in the as-polished condition achieved indexing hit rates of around $80 \%$. Samples lightly etched but not vibropolished achieved indexing hit rates between $85-90 \%$. Etched and vibropolished samples achieved hit rates indexing as high as 95\% (FIG. 3).

References:

[1] Specimen Preparation for Electron Backscattered Diffraction, G.F. Vander Voort, William Van Geertruyden

[2] Struers E-Metalog, Non-Ferrous Metals $>$ Wrought Al Alloys

[3] ASM Handbook, Vol 9, Metallography and Microstructures, Mar. 2000, pp. 354

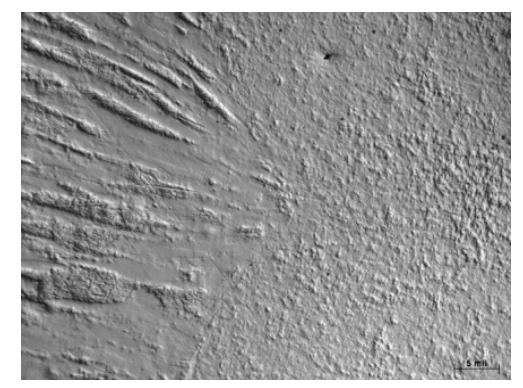

As Etched

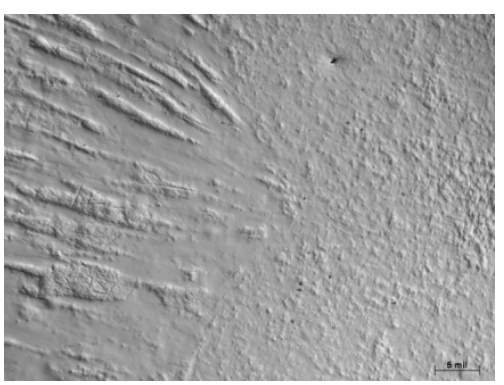

As Vibropolished 30 min

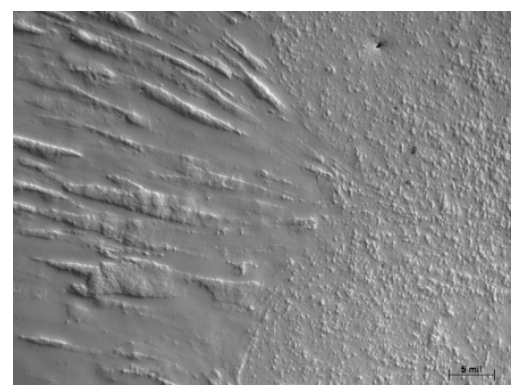

As Vibropolished $60 \mathrm{~min}$

FIG. 1. DIC Micrographs of 2195 Weld Nugget Transition W/Progressive Vibropolishing

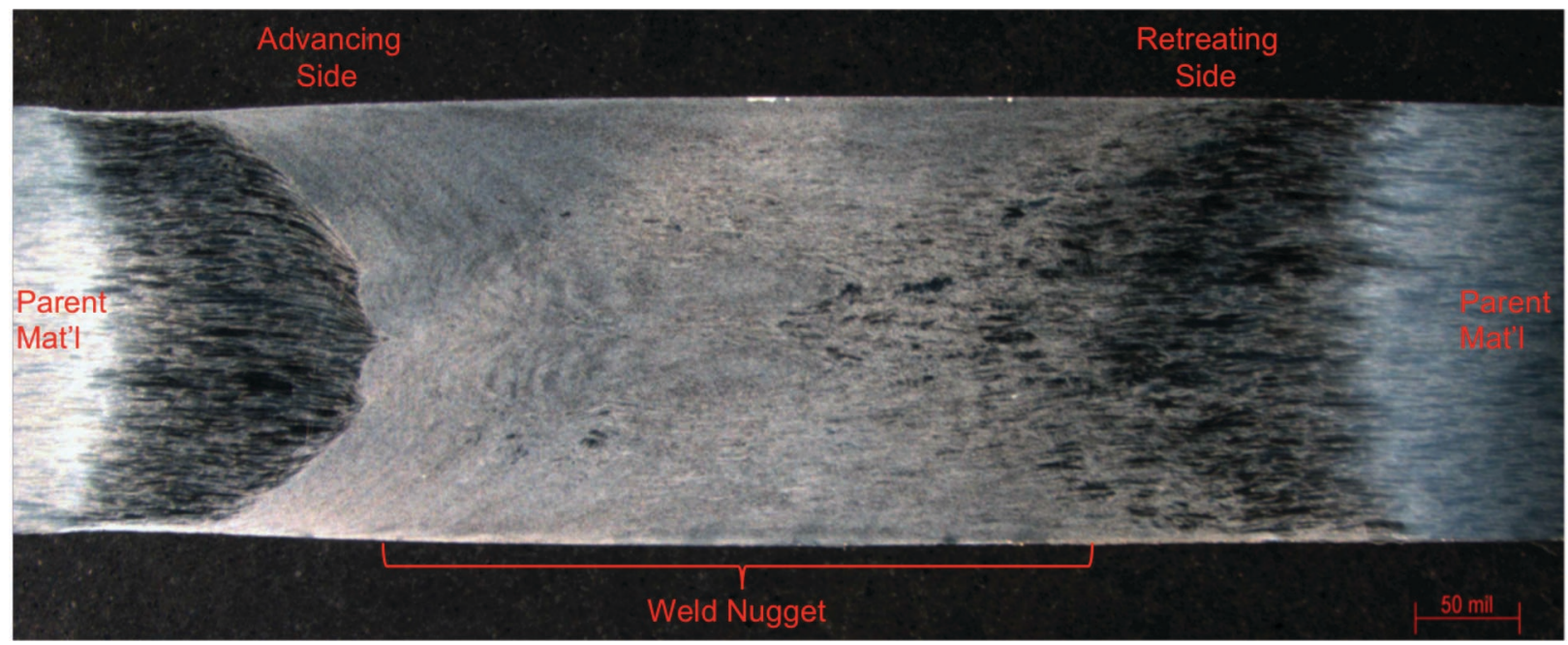

FIG. 2. Optical Micrograph of SR-FSW 2195 Al-Cu-Li Alloy

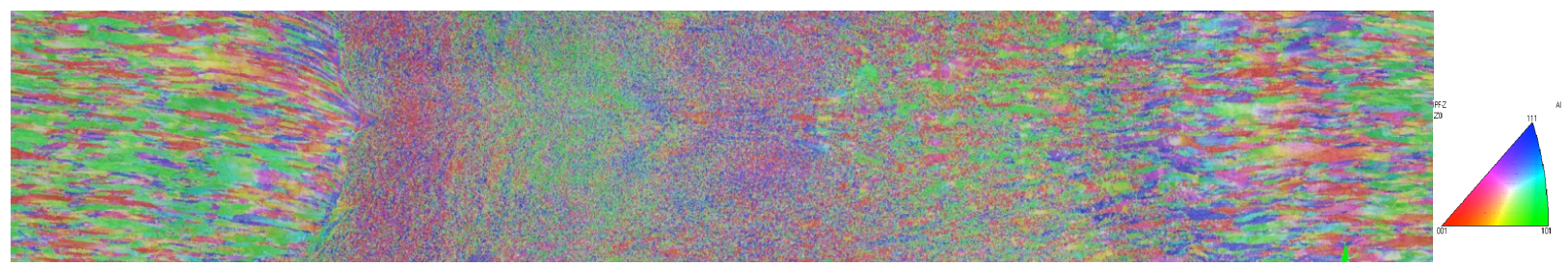

FIG. 3. EBSD Inverse Pole Figure Map of SR-FSW 2195 Al-Cu-Li Alloy 